glottic venous malformation following sclerotherapy. One patient had a corneal abrasion. 11/12 patients had improvement in the size and symptoms of the venous malformations. Conclusions Cone Beam CT/XperGuide needle guidance is a useful tool for treatment of deep seated venous malformations, particularly those around the airway. In our case series we had high rates of technical success for delivery of sclerosant to these malformations and a low complication rate.

Disclosures W. Brinjikji: 1; C; NIH/NINDS, Johnson and Johnson/Cerenovus. 2; C; Johnson and Johnson/Cerenovus, Microvention. 4; C; Marblehead Medical LLC.

\section{E-115 PTERYGOVAGINAL ARTERY: A LESS RECOGNIZED RECURRENT BRANCH FROM THE DISTAL INTERNAL MAXILLARY ARTERY}

K Yoshida*, T Akiyama. Neurosurgery, Keio University School of Medicine, Tokyo, JAPAN

\subsection{6/neurintsurg-2020-SNIS. 147}

Introduction The pterygovaginal artery (PtVA) is one of the recurrent branches from the distal part of the internal maxillary artery (IMA), which courses through the pterygovaginal canal (PtVC) that connects the pterygopalatine fossa and the nasopharynx. Less attention has been paid to this artery in spite of its unique anatomical features and important anastomoses around the eustachian tube because on angiography this minute artery can be hardly distinguished due to overlaps of many IMA branches. Nevertheless, widespread utilization of cone beam computed tomography (CBCT) has enabled us depicting even extremely small arteries. In cases with hypervascular lesions, PtVA is often enlarged and can be identified with attentive observation. We present three cases of tumor embolization before endonasal endoscopic approach and underscore its clinical implications in neurointerventional surgery.

Case 1 (Sinonasal renal cell-like adenocarcinoma) A 66-year-old woman with a history of von Hippel-Lindau disease and multiple surgeries for intracranial hemangioblastomas was scheduled for a resection of an enlarging mass extending around the ethmoid and sphenoid sinuses. Along with branches from the left sphenopalatine artery (SPA), the left PtVA, branching from the most distal part of the IMA, was embolized selectively with $40 \%$ n-butyl cyanoacrylate.

Case 2 (Sphenoid Sinus Hemangioma) A 20-year-old man presented with the right abducens nerve palsy. MRI showed an enhancing mass around the sphenoid sinus extending to the cavernous sinus. CBCT revealed that the main feeder from the IMA was the PtVA, branching from a relatively proximal portion of IMA, lateral to the pterygomaxillary fissure. The selective angiography from the PtVA showed a tumor stain and a branch to the nasopharyngeal roof anastomosing with the ascending pharyngeal artery (APhA) and ascending palatine artery. After the microcatheter was further advanced close to the lesion, embospheres of $300-500 \mu \mathrm{m}$ were cautiously injected and platinum coils were deployed in the PtVA. Postembolization CBCT showed coils in the PtVC.

Case 3 (Pituicytoma) A 69-year-old woman with a large sellar tumor underwent a preoperative embolization. The distal IMA, middle meningeal arteries, accessory meningeal artery, APhAs were embolized with coils. The tumor stain from the distal IMA was specifically derived from the PtVA, the Vidian artery, and the artery of the foramen rotundum.

Conclusion The PtVA has a medial or lateral origin from the distal IMA and anatomical knowledge for this artery is essential in neurointervention. Embolization should be performed with precaution to avoid unintended migration through dangerous anastomoses.

Disclosures K. Yoshida: None. T. Akiyama: None.

\section{E-116 LONG-TERM MORTALITY AFTER CAROTID STENTING}

${ }^{1} \mathrm{~L}$ Rinaldo*, ${ }^{2} \mathrm{~A}$ Bhargav, ${ }^{3} \mathrm{C}$ Arnold Fiebelkorn, ${ }^{1} \mathrm{G}$ Lanzino. ${ }^{1}$ Neurosurgery, Mayo Clinic, Rochester, MN; ${ }^{2}$ Mayo Clinic Alix School of Medicine, Mayo Clinic, Rochester, MN; ${ }^{3}$ Neurology, Mayo Clinic, Rochester, MN

\subsection{6/neurintsurg-2020-SNIS.148}

Background Carotid artery stenting (CAS) is an established procedure for the treatment of atherosclerotic disease affecting the extracranial internal carotid artery. Recent populationbased studies have suggested that long-term survival after CAS may be limited, thereby questioning its efficacy in a real-world scenario.

Methods We retrospectively reviewed outcomes of patients undergoing CAS for asymptomatic or symptomatic carotid stenosis by a neurosurgeon or interventional neuroradiologist at our institution between 2008 and 2018. Patient and disease characteristics were recorded, as was the incidence of peri-procedural and overall ischemia and mortality after CAS. Risk factors for recurrent ischemia and mortality were identified using a Cox proportional hazards model.

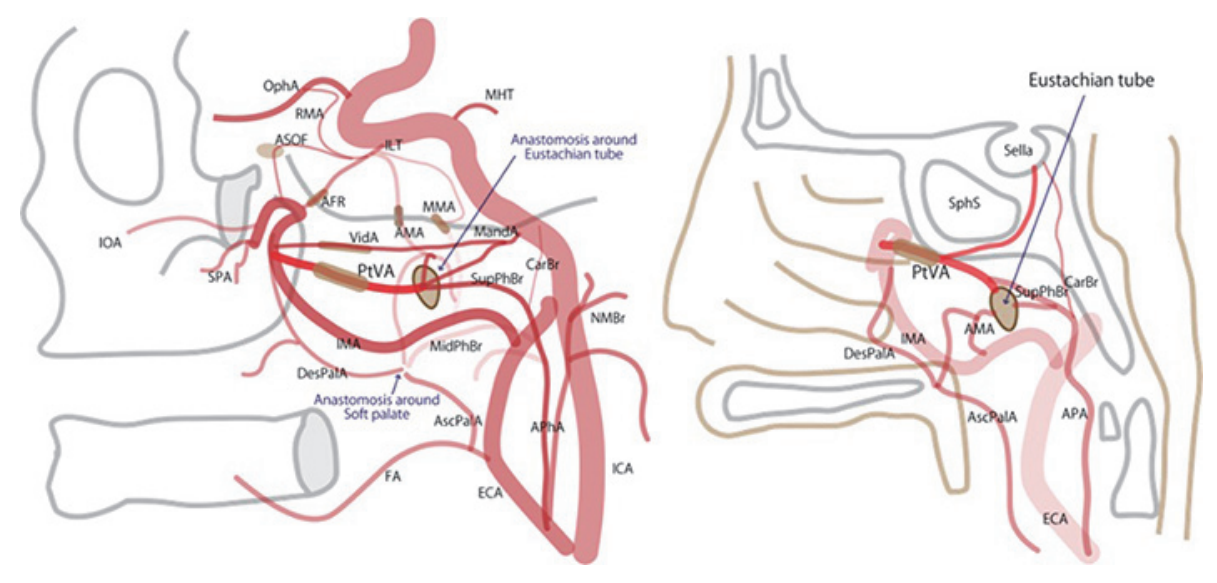

